\section{More Than One Ever Wanted To Know About X-ray Detectors}

\author{
Part 2 - Settling the question of detector warming \\ Mark W. Lund, MOXTEK, Inc.
}

A combination of electron microscope and $x$-ray spectrometer is a very powerful tool. Not only can one see a sample in great detail, but one can determine, and even map, the chemical elements. In Part 1, I discussed some of the basics of energy dispersive x-ray spectroscopy (EDS or EDX). The heart of the spectrometer is a small piece of single crystal silicon about the size and shape of a shirt button, and about twice as thick. It has been selected for high purity, and then lithium drifted to compensate the remaining impurities.

The lithium is carefully drifted into the crystal button in order to exactly compensate the impurities in the crystal that would create leakage current. This is done at about $60^{\circ} \mathrm{C}$ under an electric field. It is then evaluated and re-drifted for a final clean up of any uncompensated impurity atoms that remain. These processes are very sensitive and the exact details are trade secrets in every case. After the lithium drifting is finished the crystal is fabricated into a detector by applying electrodes and passivating its surfaces. The finished detector is then installed in its Dewar, cooled to liquid nitrogen temperature and tested for final performance.

The finished detector is then kept at that cooled temperature for the rest of its useful life. Allowing the detector to warm up will allow the lithium to drift and ruin the detector.

Actually, the last sentence is not completely true. Certainly in the early years of Si(Li) detectors the wise ones kept them cold. Warming the detector not only encouraged the lithium to drift, but also cracked the passivation on the detector surfaces. However, this was not what the customer wanted to buy, and airlines complained about transporting this bomb-shaped object with white smoke pouring out of it. As the technology improved, it became possible to make detectors out of increasingly better silicon, which requires much less lithium. Passivation techniques were also developed which increased the stability of the detectors. A modern detector can be warmed and cooled hundreds of times without deteriorating.

Actually, the last sentence is not completely tue either. While it is tue that modern $\mathrm{Si}(\mathrm{Li})$ detectors will not be damaged by warming and cooling, there are other issues to be considered.

The spectrometer is constructed from a number of different materials including silicon, copper, aluminum, boron nitride, gold, Kovar, etc. - each with a different thermal coefficient of expansion. The heating and cooling of the spectrometer, over a range of some $220^{\circ} \mathrm{C}$, will inevitably cause some "creaks" and "groans." These may have the effect of moving the contacts, and inducing scratches which may cause the detector surfaces to produce more noise. Thermal cycling will also stress other parts of the detector package. So...if you have an old detector DON'T WARM IT UP! If you have a modern detector you may warm it up occasionally without fear - . - but DON'T DO IT A LOT! And that is the truth (or at least good advice)!

Next I would like to talk about one of my heros: Dr. U. Fano. Many people think that "fano" is another word for "fudge." as in fudge factor and others actually blame Dr. Fano for limiting detector resolution. He was, however, a pioneer in understanding the absorption of ionizing radiation. His influence in the EDX world is in the mathematical factor " $F$." While he did not name the factor, perhaps out of modesty, he did give it his initial. Others later took the hint and called it the Fano factor.

An energy dispersive detector works by absorbing an $x$-ray into the silicon crystal, and measuring the charge created in the crystal by the $x$-ray. This works best if all $x$-rays of a certain energy produce exactly the same number of electron-hole pairs in the detector. If they don't, there will be some broadening of the $x$-ray peak, giving a fundamental limit to detector resolution. You have already guessed that they don't, there is broadening, and there is a fundamental limit. These fluctuations are due to the quantum nature of the charge creation process. To understand how Dr. Fano comes in, we will go into a brief (and hopefully entertaining) discussion of statistics.
Say that you are camping in Yosemite and notice that every time you slap your knee you kill a hand full of mosquitos. By doing a controlled experiment you notice that by slapping four times a minute you kill on the average 100 mosquitos per slap. But you won't kill 100 mosquitos every slap, because the mosquitos don't fly in packs of 100, they just show up one at a time at random. Because the mosquito arrivals are independent you will find that the standard deviation in knee-kill-counts is the square root of the average number killed, i.e. 10. After a couple of hours, if you plot a histogram of the data, you will find a peak of 100 and a full width at half max. at $2.4^{\star} 10$, or 24 ( 2.4 converts standard deviation to full width half max.). Such independent processes are called Poisson statistics, which for reasonably large counts are approximated by Gaussian statistics.

Luckily for us, the generation of charge in a Si(Li) detector does not obey Poisson statistics. If it did, the statistical limit for resolution at FE-55 (Mn Ko) would be $350 \mathrm{eV}$, which is well over twice what the best Si(Li)'s actually achieve. The reason that charge creation in $\mathrm{Si}(\mathrm{Li})$ detectors does not follow Poisson statistics is that the generation of electron-hole pairs by an $x$-ray is not a series of independent events. This was discovered during World War $I$ and was published by Dr. Fano in 1946 and 1947.

The difference between Poisson statistics and Fano statistics is just a constant (actually the square root of a constant), called the Fano factor $(F)$. The Fano factor cannot yet be accurately calculated from theoretical considerations and it is very hard to measure. The best measurements have shown that the Fano factor for silicon is no larger than about 0.08 . Thus the statistical limit of resolution is 3.5 times better than we would get without Fano (remember that $F$ is under the square root).

So, the next time you hear a sales person say "our resolution is limited primarily by the Fano factor" you can tell him that his resolution is limited by quantum statistics, and that the Fano factor is increasing his resolution, saving his bacon, and feeding his children. Or you can just keep quietly smug.

All right, if the fundamental limit of resolution is $100 \mathrm{eV}$, why are the best Si(Li) detectors at around $130 \mathrm{eV}$ ? The reason is that other factors influence resolution including noise from leakage current, sufface noise, incomplete charge collection, and preamplifier noise. I will discuss some of these fascinating topics in this column in the future.

A crystal with a lower band gap than silicon (i.e. germanium) will produce more charge than silicon. This will produce less statistical noise, which is the reason that the new germanium detectors have better resolution than with silicon. I will also discuss germanium detectors in this column in the future

\section{More on Projection Slides \\ Theodore M. Clark, J। Case}

The June/July issue of this publication contains an article by $\mathrm{A}$. Kent Christensen on how the $10 \mathrm{X}$ increase in price of Kodak $2^{\prime \prime} \times 2^{\prime \prime}$ Projector Slide Plates now prices this method of making projection slides from black and white negatives beyond the reach of most electron microscopists. Kent comments that perhaps the EM negatives can be printed on sheet film to obtain wide tonal range projection slides

I have had very good success using Kodak 5302 Positive Release film to make $35 \mathrm{~mm}$ slides from 35 negatives. I use a condenser enlarger and project into a $35 \mathrm{~mm}$ camera held in a special angle plate $/$ constructed. This $35 \mathrm{~mm}$ film is blue sensitive, extremely fine grained, 125 lines $/ \mathrm{mm}$ resolution, ASA4 with tungsten lighting, and available at low cost in $100 \mathrm{ft}$. rolls. The film is easily developed in a tank using print developer, fixing, and rinsing. I am told that this film dates back to the 1920's and was intended for making positive copies of movie film black and white negative originals. My Inter/Micro ' 84 and ' 86 presentations used slides made with 5302 .

If one wants slides that have resolution closely approaching that of the original negatives, it is of great importance to use a macro lens with optimum correction for the magnification used in the copy process and at nearly full aperture for maximum resolution. My experience with 5302 is described in greater detail in: Clark, T.M., Method for Calculating Relative Apertures for Optimizing Diffraction Limited Depth of Field in Photomacrography, The Microscope, 32, 219-258 (1984) 